\title{
Risk Factors Screening for Gestational Diabetes Mellitus Heterogeneity in Chinese Pregnant Women: A Case-Control Study
}

This article was published in the following Dove Press journal: Diabetes, Metabolic Syndrome and Obesity: Targets and Therapy

\author{
Ning Wangl,* \\ Yanqi Peng ${ }^{1} *$ \\ Lu Wang' \\ Lin Song ${ }^{2}$ \\ Bo Sun ${ }^{2}$ \\ Junxiang $\mathrm{Wei}^{3}$ \\ Ting Wang ${ }^{4}$ \\ Yang $\mathrm{Mi}^{3}$ \\ Wei Cui (D)' \\ 'Department of Endocrinology and \\ Second Department of Geriatrics, The \\ First Affiliated Hospital of Xi'an Jiaotong \\ University, Xi'an, People's Republic of \\ China; ${ }^{2}$ Department of Physiology and \\ Pathophysiology, School of Basic Medical \\ Sciences, Xi'an Jiaotong University Health \\ Science Center, Xi'an, People's Republic \\ of China; ${ }^{3}$ The Second Department of \\ Obstetrics, Northwest Women and \\ Children's Hospital, Xi'an, People's \\ Republic of China; ${ }^{4}$ Department of \\ Respiratory Medicine, Xi'an No.4 \\ Hospital, Xi'an, People's Republic of \\ China
}

*These authors contributed equally to this work

Correspondence: Yang Mi

The Second Department of Obstetrics, Northwest Women and Children's Hospital, 1616 Yanxiang Road, Xi'an, Shaanxi, 710065, People's Republic of China

Tel +86-13700222I72

Email miyangmm@163.com

Wei Cui

Department of Endocrinology and Second Department of Geriatrics, The First Affiliated Hospital of Xi'an Jiaotong University, 277 West Yanta Road, Xi'an, Shaanxi, 71006I, People's Republic of China

Tel +86-1360928I695

Email doctorweiweixjtu@I26.com
Purpose: To study the risk factors of gestational diabetes mellitus (GDM) heterogeneity, and to evaluate the correlation between the risk factors and obesity.

Methods: We performed a case-control study of 452 women with GDM and 516 women with normal glucose tolerance (NGT) at the first and second trimester. We defined GDM women as GDM-resistance subtype, GDM-dysfunction subtype, and GDM-mixed subtype, according to their simultaneous insulin-release test with predominant insulin-sensitivity defect, insulin-secretion defect, or both defects.

Results: We found that higher maternal age, family history of diabetes, the elevated level of fasting blood glucose in the first trimester $(\geq 5.1 \mathrm{mmol} / \mathrm{L})$ were risk factors of all GDM subtypes. Pre-pregnancy overweight/obesity and the increased gestational weight gain (GWG) in the first-trimester are risk factors of the GDM-resistance subtype. Indicators including younger age at first menstruation, the elevated levels of alanine aminotransferase (ALT), total bile acid (TBA), triglyceride (TG), and the decreased level of high-density lipoprotein cholesterol (HDL-C) are risk factors of the GDM-resistance subtype. However, the associations between those risk factors and GDM-resistance subtype attenuated after adjusted by pre-pregnancy body mass index (pre-BMI) and gestational weight gain (GWG) in the first trimester. Nonalcoholic fatty liver disease (NAFLD) and the improved level of TG are independent risk factors for the GDM-resistance subtype and the GDM-mixed subtype, respectively.

Conclusion: Women with GDM exhibited heterogeneity based on glycemic physiology and their risk factors are not all the same. Some obesity-related risk factors are specific to the GDM-resistance subtype, which are mediated by pre-pregnancy overweight/obesity and the elevated GWG the first-trimester.

Keywords: pregnancy, pre-pregnancy BMI, gestational weight gain, obesity, insulin resistance, insulin secretion

\section{Introduction}

Gestational diabetes mellitus (GDM) is a common complication of pregnancy period in which spontaneous hyperglycemia usually happens during the second and third trimesters without known diabetes before pregnancy. ${ }^{1}$ GDM increasing the risks for adverse perinatal outcomes such as metabolic disease for both mother and infants. ${ }^{2}$ Insufficient insulin secretion and decreased insulin sensitivity have been verified as the two predominant drivers of hyperglycemia in non-pregnant diabetes individuals. ${ }^{3}$ Powe $^{4}$ first observed and reported that heterogeneity existed in the physiologic and pathologic processes leading to hyperglycemia in women 
with GDM. GDM heterogeneity refers to the variety in genetic factors, racial and demographic differences, betacell function, and some special clinical characteristics. In the current study, we specifically targeted on the heterogeneity of insulin sensitivity and beta-cell function and the pathogenesis of GDM, with our subtypes described as GDM with a predominant insulin-secretion defect, GDM with a predominant insulin-sensitivity defect, or GDM with both defects.

Many studies focus on risk factors of GDM, but these results lack consistency. A meta-analysis of European, North American and Australian cohorts found that maternal pre-pregnancy overweight/obesity is associated with the risk of GDM, ${ }^{5}$ while another study uncovered that the effect of pre-pregnancy body mass index (pre-BMI) as a screening characteristic to predict GDM varied by race/ethnicity. ${ }^{6}$ The effect of other reported risk factors on GDM such as maternal lipid profile and different diet/ lifestyle changed according to BMI. ${ }^{7-9}$ Variations in population and diagnostic criteria for GDM partly account for the differences in these findings. The heterogeneity of physiologic hyperglycemia among women with GDM may explain this inconsistency from a new perspective. Our previous research found that GDM women of the three subtypes had different clinical characteristics, ${ }^{10}$ and we speculate that the discrepancy of risk factors also existed among the three GDM subtypes.

Obesity causes a systemic inflammatory response, with possible downstream metabolic sequelae, including insulin resistance and glucose dysregulation. ${ }^{11}$ Some studies used stratified BMI, lean/obese phenotypes to determine risk factors of developing GDM, ${ }^{9,12}$ however, none of these researches reflected the causes of hyperglycemia in pregnant women.

Recently, many studies uncovered some new risk factors of GDM, such as the increased level of total bile acid $(\mathrm{TBA})^{13}$ and ferritin, ${ }^{14}$ nonalcoholic fatty liver disease $(\mathrm{NAFLD})^{15}$ and thyroid dysfunction ${ }^{16}$ at the 1 st trimester, earlier age at menarche, ${ }^{17}$ and persistent vitamin D deficiency. ${ }^{18}$ However, few studies analyzed the influence of these new risk factors on GDM according to the heterogeneity of physiologic hyperglycemia.

Since the heterogeneity could reflect the pathophysiologic state of GDM and the different clinical manifestations more objectively, we carried out a case-control study research to elucidate the variations in risk factors of the GDM subtypes, and to evaluate the correlation between GDM subtypes and obesity.

\section{Patients and Methods}

\section{Study Population and Design}

We performed a case-control study, and data were collected from Han Chinese women underwent routine gestational care visit at the Department of Obstetrics of the Northwest Women and Children's Hospital from November 2019 to March 2020. We collected clinical data at different follow-up times (1st-trimester study visit at 12 weeks of gestation, 2nd-trimester study visit at 24-28 weeks of gestation). Woman underwent the 75-g, 2-h oral glucose tolerance test (OGTT) at the 24th-28th gestational week, and GDM was diagnosed according to the International Association of Diabetes and Pregnancy Study Group (IADPSG) criteria $^{19}$ were included. The inclusion criteria is women of bearing age with full-term fetus and complete pregnancy data. From a total of 3829 women, the data of 1204 women who underwent OGTT were collected. The exclusion criteria included that: a history of diabetes $(\mathrm{n}=12)$; OGTT results showed a fasting glucose $\geq 7.0 \mathrm{mmol} / \mathrm{L}$, 2-hour post-meal blood glucose was $\geq 11.1 \mathrm{mmol} / \mathrm{L}$, or glycated hemoglobin Alc (HbAlc) was $\geq 6.5 \%$ in the 1 st trimester $(n=25)$; if they showed preeclampsia $(\mathrm{n}=31)$, younger than 18 years of age $(\mathrm{n}=35)$; had multiple gestations $(\mathrm{n}=7)$, maternal disease $(n=23)$, or missing vital data $(n=23)$. Finally, 1048 subjects were eligible and included in further analysis.

\section{Clinical Measurements and Definitions}

The recorded weight and height at the first gestational care visit were used to calculate pre-BMI. Pre-BMI was categorized according to criteria specific for Chinese adults (BMI $<18.5 \mathrm{~kg} / \mathrm{m}^{2}$, emaciated; BMI, $18.5-23.9 \mathrm{~kg} / \mathrm{m}^{2}$, normal weight; BMI, 24-27.9 kg/m $\mathrm{m}^{2}$, overweight; and BMI > $28 \mathrm{~kg} / \mathrm{m}^{2}$, obese). ${ }^{20}$ Gestational weight gain $(\mathrm{GWG})$ in the first-trimester was calculated as the maternal weight at the second-trimester visit minus pre-pregnancy body weight at the first gestational care. The presence or absence of NAFLD was evaluated utilizing liver ultrasound, and it was defined as the detection of bright echogenic patterns within the liver detected by ultrasonography. The Insulin Sensitivity Composite Index (ISI composite index) and the Stumvoll I index were used to measure insulin sensitivity and secretion according to the data from the OGTT and simultaneous insulin-release test. ${ }^{21-23}$ We defined GDM subtypes using Powe's classification ${ }^{4}$ according to the distributions of insulin sensitivity and secretion in women compared with the 
normal glucose tolerance (NGT) group. We considered women with GDM to have an insulin secretion defect or sensitivity defect if insulin secretion or sensitivity was below the 25 th percentile. According to the above standards, women with GDM were classified into the following subtypes: GDM with a predominant insulin-sensitivity defect (GDM-resistance, $\mathrm{n}=202$ ), GDM with a predominant insulin-secretion defect (GDM-dysfunction, $n=140$ ), or GDM with both of the above-mentioned traits (GDM-mixed, $n=$ 110). We excluded participants with GDM who had both insulin sensitivity and insulin secretion above the 25 th percentile $(\mathrm{n}=77)$, and women who were treated with insulin $(\mathrm{n}=3)$.

\section{Data Collection and Laboratory Measurements}

The data included a questionnaire for pregnant women contained general information regarding their present and past medical histories, family history of diabetes, reproductive history, medication history, alcohol consumption, smoking status, and age at first menstruation. Fasting blood glucose (FBG) were tested using the glucose oxidase method (intra-assay and inter-assay coefficients of variation were $2.1 \%$ and $2.6 \%$, respectively), highperformance liquid chromatography was used to measure hemoglobin A1c (HbA1c) percentage (Skylight Biotech, Akita, Japan). Reagents, which were provided by Chinese Shandong 3V Bioengineering Company, were used to test aspartate aminotransferase (AST), alanine aminotransferase (ALT) and TBA, and the detection was performed with the Hitachi 7600 automatic 49-biochemical analyzer. The enzymatic method (Parsazmun, Karaj, Iran) was used to detect lipid profiles [serum triglycerides, total cholesterol, low-density lipoprotein cholesterol (LDL-C) and high-density lipoprotein cholesterol (HDL-C) concentrations]. Serum 25-hydroxyvitamin $\mathrm{D}_{3} \quad[25-(\mathrm{OH})$ $\mathrm{D}_{3}$ ] was analyzed using the electro chemiluminescent immunoassay on Cobas e411 Elecsys 2010 (Roche Diagnostics $\mathrm{GmbH}$, ranged 4-100 ng/mL). Serum insulin and ferritin levels were tested by electrochemiluminescent immunoassay kits of Chinese Shandong $3 \mathrm{~V}$ Bioengineering Company (insulin, R-C-01-01, ranged 5-180 $\mu \mathrm{U} / \mathrm{mL}$; ferritin, R-B-05-01, ranged 0-500ng/mL). Thyroid peroxidase antibodies (TPOAb) were measured by the radioimmunoassay of Chinese Shandong $3 \mathrm{~V}$ Bioengineering Company (R-A-08-01, ranged 0.1$1000 \mathrm{IU} / \mathrm{mL}$ ). All the laboratory tests are performed by the standard laboratory methods in the certified laboratory of the Northwestern Women's and Children's Hospital in Xi'an, China. The ISI composite index = $10,000 / \sqrt{ }($ fasting glucose $\times$ fasting insulin $) \times($ mean glucose $\times$ mean insulin), ${ }^{21}$ using glucose and insulin measurements expressed as $\mathrm{mmol} / \mathrm{L}$ and $\mathrm{uU} / \mathrm{mL}$, respectively. The Stumvoll I index $=2032+4.681 \times$ Ins0$135.0 \times$ Gluc $120+0.995 \times$ Ins $120+27.99 \times$ BMI-269.1 $\times$ Gluc$0,{ }^{23}$ using glucose and insulin measurement expressed as $\mathrm{mmol} / \mathrm{L}$ and $\mathrm{uU} / \mathrm{mL}$, respectively. The homeostasis model assessments (HOMA2-S and HOMA2- $\beta$ ) at http://www.dtu.ox.ac.uk (accessed on 11 January 2016) were used as indices to evaluate insulin sensitivity and secretion function, respectively.

\section{Statistical Analysis}

We used SPSS Statistics 22.0 (IBM SPSS, Chicago, IL, USA) to analyze our data. Data are expressed as means (standard deviation, SD) or median (interquartile range, IQR). Differences across the four groups (NGT and three GDM subtypes) were compared using one-way ANOVA for normally distributed continuous variables, the Kruskal-Wallis test for non-normally distributed continuous variables, and the Chi-squared test (or Fisher's exact-probability test) for categorical variables. When the $P$-value from the ANOVA, Kruskal-Wallis test, or Chi-squared test (or Fisher's exact test) was $<0.05$, pairwise comparisons between the NGT group and each GDM subgroup were made using the Tukey's test, Dunn's test, Chi-squared test, respectively. $P$-values for Chi-squared test pairwise comparisons were adjusted using the Bonferroni correction. Then, two adjusted polytomous logistic regression models were fit to identify potential risk factors of GDM-subtypes. The first one was adjusted for each other, alcohol consumption, and smoking status; the second included the first model plus pre-BMI and GWG in the first trimester. The reference value was the NGT group. The goodness of fit was tested using the Hosmer-Lemeshow test.

\section{Results}

\section{Baseline Data}

Baseline data are shown in Table 1. From a total of 3829 women, 1204 women underwent the oral $75 \mathrm{~g}$ glucose tolerance test (OGTT), and 532 of them (13.9\%) were diagnosed with GDM. Among women with GDM, 202 patients $(38.0 \%)$ were classified into the GDM-resistance 
Table I Characteristics of GDM Subtypes and NGT Group

\begin{tabular}{|c|c|c|c|c|c|c|c|}
\hline & $\begin{array}{c}\text { GDM- } \\
\text { Resistance }\end{array}$ & $P^{\mathbf{a}}$ & $\begin{array}{c}\text { GDM- } \\
\text { Dysfunction }\end{array}$ & $P^{\mathbf{a}}$ & GDM-Mixed & $P^{\mathbf{a}}$ & NGT \\
\hline Number & 202 & & 140 & & 110 & & 516 \\
\hline Maternal age (years) & $31(28-34)$ & 0.019 & $31(29-34)$ & 0.010 & $31(28-33)$ & 0.018 & $30(28-33)$ \\
\hline Smoking (n, \%) & $7(3.5)$ & - & $4(2.9)$ & - & $0(0)$ & - & $13(2.5)$ \\
\hline Alcohol use (n, \%) & $3(1.5)$ & - & $2(1.4)$ & - & $3(2.7)$ & - & $9(1.7)$ \\
\hline $\begin{array}{l}\text { Family history of diabetes mellitus } \\
(n, \%)\end{array}$ & $36(17.8)$ & $<0.001$ & $19(13.6)$ & 0.001 & $14(11.8)$ & 0.004 & $27(5.23)$ \\
\hline Nulliparous (n, \%) & III (54.9) & - & $82(58.6)$ & - & $64(58.2)$ & - & $303(58.7)$ \\
\hline $\begin{array}{l}\text { History of macrosomia at delivery } \\
(n, \%)\end{array}$ & II (5.4) & - & $7(5.0 \%)$ & - & $3(2.7)$ & - & $16(3.1)$ \\
\hline Pre-BMI $\left(\mathrm{kg} / \mathrm{m}^{2}\right)$ & $\begin{array}{c}23.73 \\
(20.38-26.42)\end{array}$ & $<0.001$ & $\begin{array}{c}21.91 \\
(18.35-24.83)\end{array}$ & 0.213 & $\begin{array}{c}20.60 \\
(18.34-23.14)\end{array}$ & 0.903 & $\begin{array}{c}22.68 \\
(20.53-24.50)\end{array}$ \\
\hline Height $(\mathrm{cm})$ & $162.36 \pm 4.74$ & - & $161.43 \pm 5.30$ & - & $161.53 \pm 4.74$ & - & $161.83 \pm 4.70$ \\
\hline Age at first menstruation (year) & $13.0(\mid 1.0-15.0)$ & $<0.001$ & $15.0(13.75-15.0)$ & 0.982 & $14.0(14.0-16.0)$ & 0.985 & $14.0(13.75-15.0)$ \\
\hline
\end{tabular}

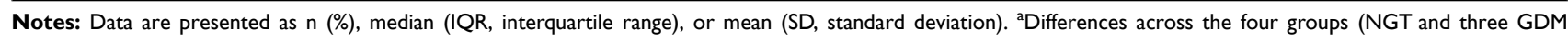
subtypes) were compared using one-way ANOVA for normally distributed continuous variables, Kruskal-Wallis test for non-normally distributed continuous variables, or Chi-squared test (or Fisher's exact test) for categorical variables. When $P<0.05$, pairwise comparisons between the NGT group and each GDM group were made using the Turkey's test, Dunn's test, Chi-squared test, or Fisher's exact test, respectively. P-values for pairwise comparisons were adjusted using the Bonferroni correction. Abbreviations: GDM, gestational diabetes mellitus; NGT, normal glucose tolerance; pre-BMI, pre-pregnancy body mass index.

group, 140 patients $(26.3 \%)$ were assigned to the GDMdysfunction group, and 110 patients $(20.7 \%)$ were classified into the GDM-mixed group. All women in the three GDM subtypes exhibited an elder maternal age $(P=0.019$, $P=0.010$, and $P=0.018$, Table 1$)$, and a higher percentage of family diabetes history $(P<0.001, P=0.001$, and $P=0.004)$ compared to NGT group. The GDM-resistance group showed an elevated pre-BMI [23.73 (20.38-26.42) vs 22.68 (20.53-24.50), $\mathrm{P}<0.001]$ and a younger age of first menstruation $(P<0.001)$ relative to the NGT group, while women in the GDM-dysfunction and GDM-mixed group had pre-BMI comparable to the NGT group. Smoking status, alcohol consumption, percentage of macrosomia at delivery history, percentage of nulliparous women, and height showed no significant differences among the four groups.

\section{Indicators at the First and Second Gestational Period}

In their first trimester, women in the three GDM-subtype groups had higher plasma levels of FBG (all $P<0.001$, Table 2), while only the GDM-dysfunction group exhibited higher HbA1c $(P=0.028)$ compared with the NGT group. Indicators regarding $\mathrm{GWG}$ in the first trimester $(P=0.021)$, the percentage of NAFLD $(P<0.001)$, ALT $(P=0.029)$, TG $(P=0.012)$, and TBA $(P=0.009)$ showed a significant increase, while HDL-C $(P=0.024)$ demonstrated a remarkable reduction in the GDM-resistance group when compared to the NGT group. The TG values and the percentage of NAFLD in the GDM-mixed group were higher than those in the NGT group $(P=0.009$ and $P=$ 0.006). Meanwhile, 25-(OH) $\mathrm{D}_{3}$, AST, CHO, LDL-C, ferritin, and TPOAb showed no significant differences among the four groups.

In the second trimester, women in the three GDMsubtype groups manifested higher blood glucose at all the time points of the OGTT test, and larger glycemic area under the curve (AUC) compared with the NGT group ( $P<0.001$, Table 2$)$. Plasma insulin levels at all time-points and AUC (insulin) presented to a significant increase (All $P<0.001$ ) in the GDM-resistance group relative to the NGT group. However, insulin levels and AUC (insulin) were reduced in the GDM-dysfunction and GDM-mixed group (All $P<0.01$ ) compared with the NGT group (except for fasting insulin level of GDMdysfunction group). For insulin secretion indicators, the levels of HOMA2- $\beta$, Stumvoll I index were higher in the GDM-resistance group, but lower in GDM-dysfunction 


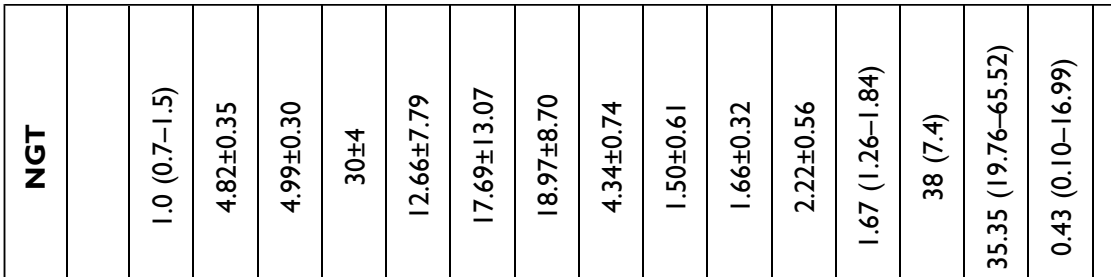

¿ I

¿্.

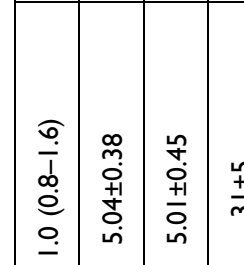

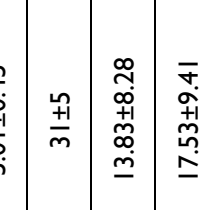

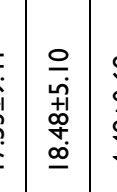

oे

๕ั

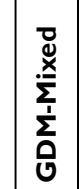

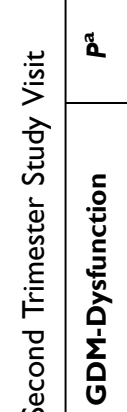

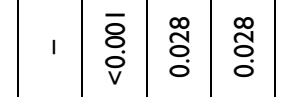

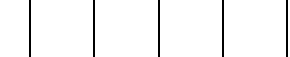

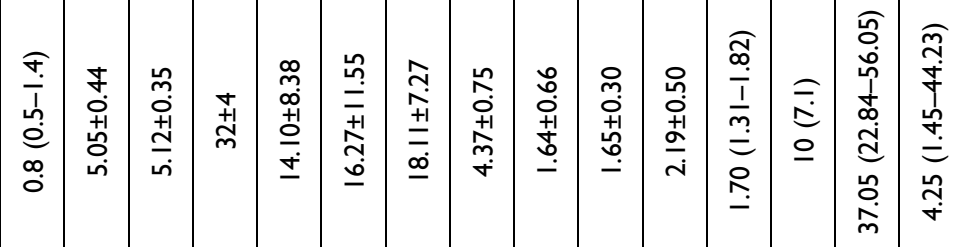

ָृ

蒙

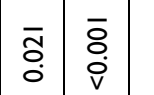

ণิ

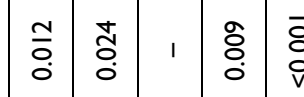

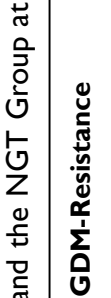

点

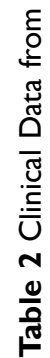

蒿

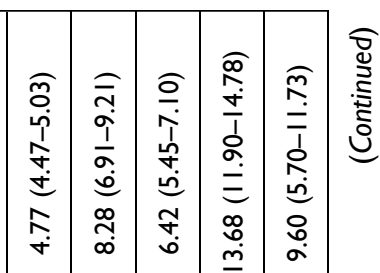

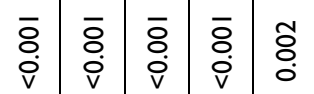

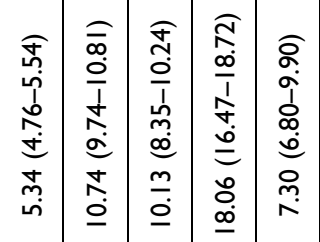

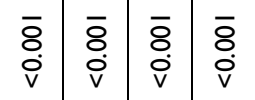

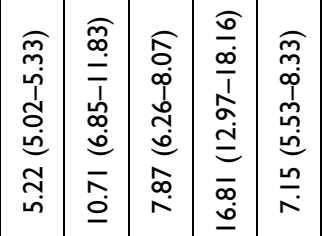

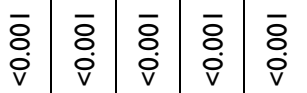

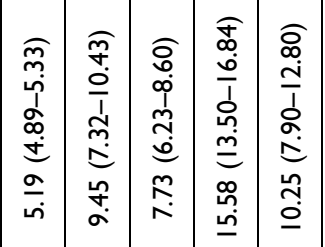

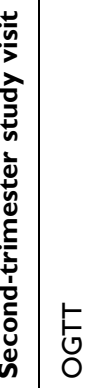




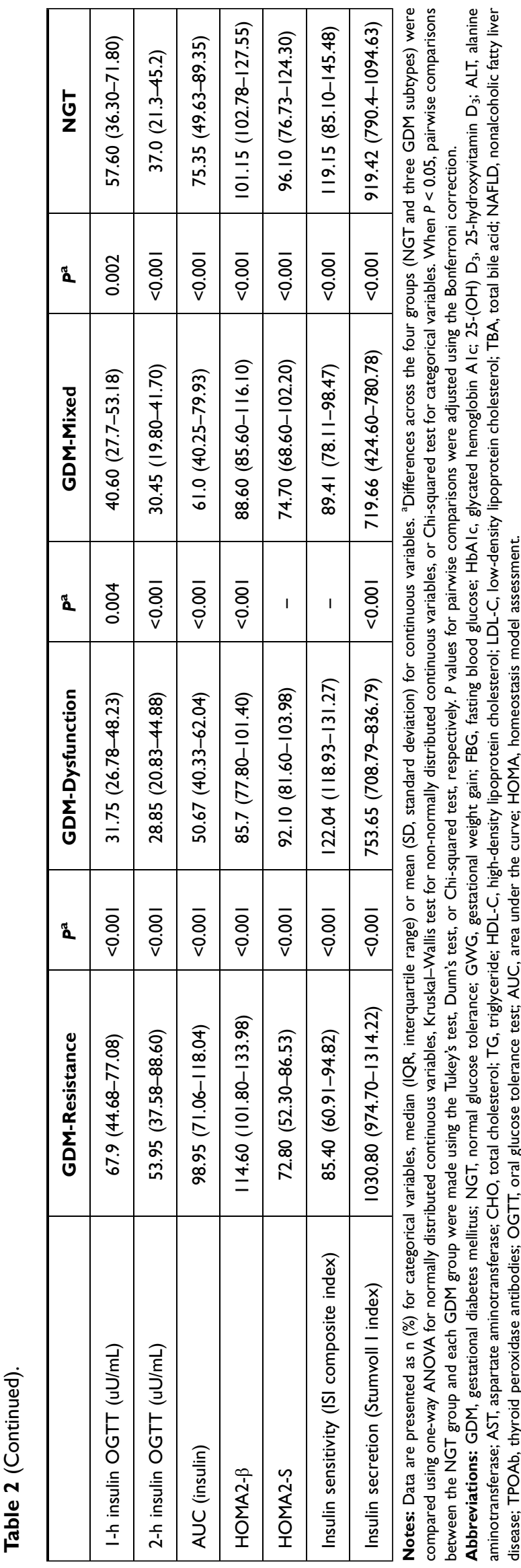

and GDM-mixed groups when compared to the NGT group (all $P<0.001$ ). Insulin sensitivity indicators (HOMA2-S and ISI composite index) were declined in the GDM-resistance and GDM-mixed groups relative to the NGT group (All $P<0.001$ ).

\section{Multivariate Polytomous Logistic Regression Analysis of Risk Factors for GDM}

In the multivariate polytomous logistic regression, the risk factors in the first trimester concerning GDM are presented in Table 3. The elevation of maternal age and the family history of diabetes are both risk factors for all GDMsubtypes compared with the NGT group (All $P<0.05$ ) in model 1 and model 2. Meanwhile, overweight/obese before pregnancy and GWG in the first trimester (All $P<0.05)$ are verified as risk factors for the GDMresistance subtype in model 2. For the indicators in plasma, increased FBG level $(\geq 5.1 \mathrm{mmol} / \mathrm{L})$ is a risk factor of all GDM subtypes regardless of whether values were adjusted or unadjusted for pre-BMI and GWG in the firsttrimester (All $P<0.05$ ). However, the increased levels of ALT, TG, TBA, and the decreased level of age at first menstruation and HDL-C are risk factors of GDMresistance subtype in model 1 (All $P<0.05$ ), but the connection between these risk factors and GDM-resistance subtype are attenuated in model 2 (model 1 plus preBMI and GWG in the first trimester as adjustments). The rising extent of TG increased the risk of GDM-mixed subtype in both model 1 and model 2 (all $P<0.05$ ). While NAFLD in the first trimester is the risk factor of the GDM-resistance group (model 1 and mode 2) and GDM-mixed group (model 1) (All $P<0.05$ ), the relationship between NAFLD and GDM-mixed subtype was diminished in model 2. The increased level of HbA1c was not a risk factor of all GDM subtypes in either model.

\section{Discussion}

Herein we implemented a case-control study in women who had GDM to investigate the risk factors of GDM subtypes that were defined by the validated indicators based on glycemic physiology.

We found that the GDM-resistance group showed higher pre-BMI and GWG in the first trimester relative to the NGT group, while women in GDM-dysfunction and GDM-mixed group had comparable characteristic above to the NGT group. Meanwhile, higher maternal age, family 


\begin{tabular}{|c|c|c|c|c|c|c|c|c|c|c|c|c|c|c|c|c|c|c|c|}
\hline \multirow{2}{*}{ 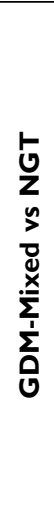 } & $\begin{array}{l}\stackrel{0}{N} \\
\overline{0} \\
\frac{0}{\Sigma} \\
\Sigma\end{array}$ & 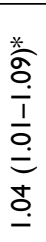 & 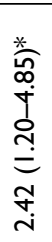 & 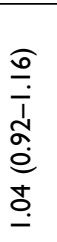 & I & 1 & 1 & 1 & 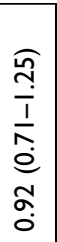 & 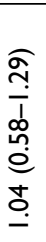 & 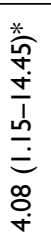 & 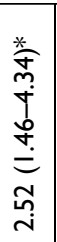 & $\begin{array}{l}\frac{a}{0} \\
\frac{1}{1} \\
\hat{\alpha} \\
0 \\
\overline{0} \\
0\end{array}$ & 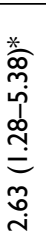 & 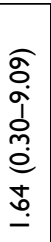 & 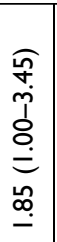 & 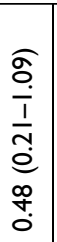 & 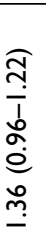 & 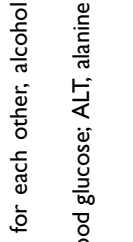 \\
\hline & $\begin{array}{l}\frac{\pi}{\bar{d}} \\
\frac{\pi}{\Sigma} \\
\frac{\delta}{\Sigma}\end{array}$ & 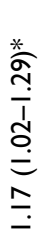 & 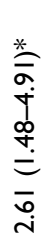 & 1 & I & 1 & 1 & 1 & & 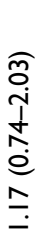 & 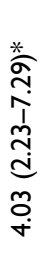 & 1 & 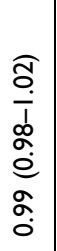 & 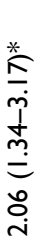 & 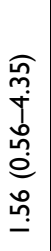 & 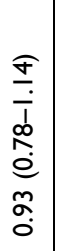 & 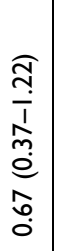 & 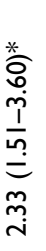 & 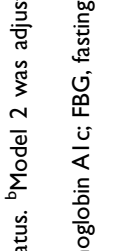 \\
\hline \multirow{2}{*}{ 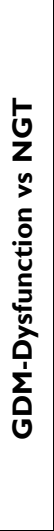 } & $\begin{array}{l}\stackrel{0}{N} \\
\bar{d} \\
\frac{0}{\Sigma} \\
\Sigma\end{array}$ & 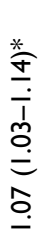 & 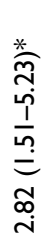 & 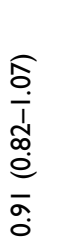 & I & 1 & 1 & 1 & $\begin{array}{l}\widehat{\sigma} \\
\infty \\
\overline{1} \\
\sigma \\
\sigma \\
0 \\
\dot{0} \\
\dot{m} \\
\underline{-}\end{array}$ & 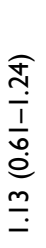 & 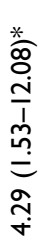 & 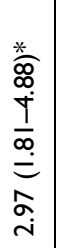 & 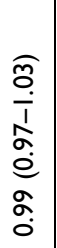 & $\begin{array}{l}\text { ô } \\
\text { în } \\
o \\
\infty \\
0 \\
0 \\
\text { ñ } \\
\end{array}$ & 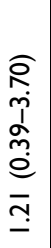 & 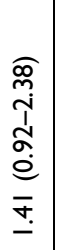 & $\begin{array}{l}\widehat{\infty} \\
\frac{1}{1} \\
\stackrel{1}{+} \\
0 \\
0 \\
0 \\
0\end{array}$ & 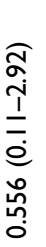 & 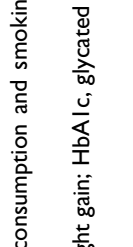 \\
\hline & $\begin{array}{l}\frac{\sigma}{\bar{d}} \\
\frac{\sigma}{\Sigma} \\
\frac{0}{\Sigma}\end{array}$ & 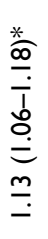 & 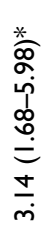 & 1 & 1 & 1 & 1 & 1 & & 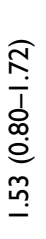 & 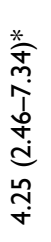 & 1 & 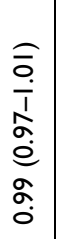 & 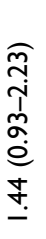 & 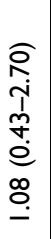 & 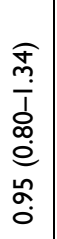 & $\begin{array}{l}\widehat{\overline{\bar{m}}} \\
\frac{1}{1} \\
\frac{1}{0} \\
\dot{0} \\
o \\
0 \\
0\end{array}$ & 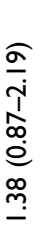 & 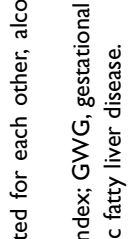 \\
\hline \multirow{2}{*}{ 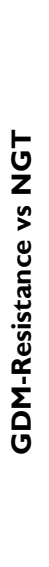 } & $\begin{array}{l}\stackrel{0}{N} \\
\overline{0} \\
\frac{0}{\Sigma} \\
\Sigma\end{array}$ & 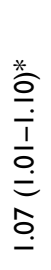 & 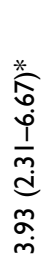 & 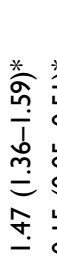 & 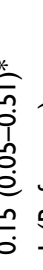 & 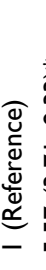 & 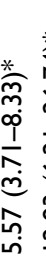 & 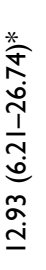 & 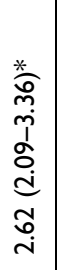 & 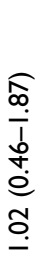 & 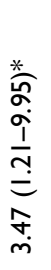 & 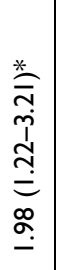 & 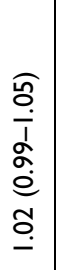 & 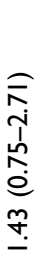 & 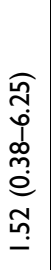 & 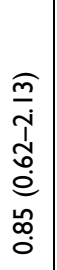 & 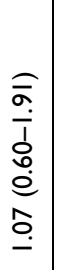 & 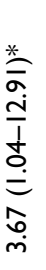 & 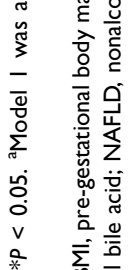 \\
\hline & $\begin{array}{l}\frac{\pi}{\bar{d}} \\
\frac{\sigma}{\Sigma} \\
\frac{\Sigma}{\Sigma}\end{array}$ & 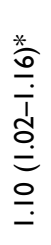 & 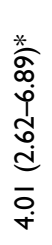 & 1 & I & 1 & 1 & 1 & & 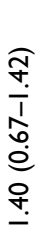 & 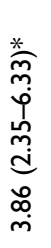 & 1 & 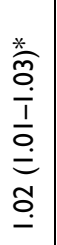 & 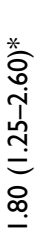 & 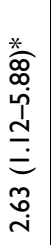 & 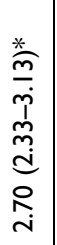 & 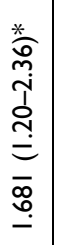 & 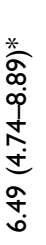 & 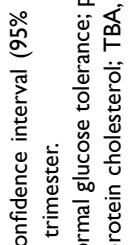 \\
\hline & & 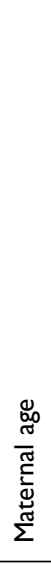 & 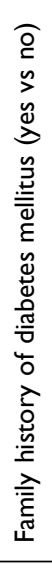 & $\begin{array}{l}\sum_{\infty} \\
\dot{d} \\
\dot{\alpha}\end{array}$ & 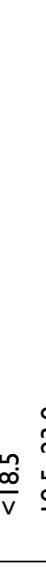 & 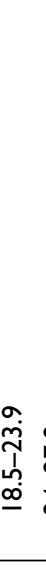 & $\frac{a}{\sim}$ & & 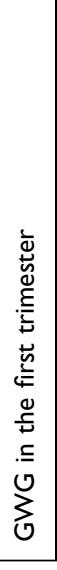 & $\begin{array}{l}\bar{\nwarrow} \\
\text { 至 }\end{array}$ & 岀 & 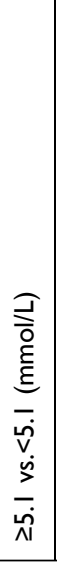 & $\varangle$ & $\vdash$ & 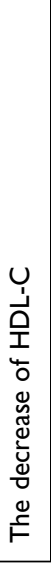 & 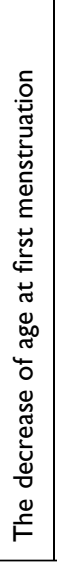 & 芯 & 号 & 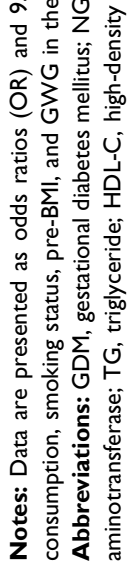 \\
\hline
\end{tabular}


history of diabetes mellitus, and the elevated level of FBG $(\geq 5.1 \mathrm{mmol} / \mathrm{L})$ in the first trimester were all risk factors of the three GDM subtypes compared with the NGT group. The increased level of TG is an independent risk factor of the GDM-mixed subtype. For the women in the GDMresistance group, overweight/obesity before pregnancy and the increased GWG in the first trimester are risk factors. Younger age at first menstruation, ascended level of ALT and TBA, and lipid metabolism disorder are risk factors of the GDM-resistance group. However, the adjustment for pre-BMI and GWG in the first trimester attenuated the observed associations. NAFLD is the independent risk factor for the GDM-resistance group regardless of whether the values were unadjusted or adjusted for pre-BMI and GWG in the first trimester.

Many studies focus on the risk factors of GDM come up with inconsistent results. ${ }^{5-9}$ There is limited research focuses on the risk factors of GDM subtypes according to the heterogeneity of physiologic hyperglycemia, and that might be one reason for this inconsistency. Many studies have verified that pre-pregnancy overweight/obesity and excess GWG during pregnancy contributes to the occurrence of GDM, ${ }^{5,24,25}$ which is consistent with our finding on the GDM-resistance subtype. We speculate the reason might be that these studies had a majority of overweight/obese participants, whose characteristic was similar to the GDM-resistance subtype in our study. Excessive fat accumulation before and during pregnancy also explains the association between the following risk factors and the GDM-resistance subtype. Some studies found that the elevated ALT level and dyslipidemia in the first trimester increasing the risk of GDM, ${ }^{26,27}$ and the higher first-trimester serum levels of TBA is also associated with $\mathrm{GDM},{ }^{13,28}$ however, the association between these risk factors and GDM are attenuated by overweight/obesity. ${ }^{26,29}$ ALT is a cytosolic enzyme that has a relatively higher concentration in the liver. When liver damage occurs, such as infection, toxins and ischemia, ALT is released into the blood from injured liver cells. On the other hand, a mild increased level of ALT within normal range usually reflect fat accumulation, which is a marker of NAFLD. ${ }^{30}$ Bile acids (BAs) are consisted of primary BAs and secondary BAs. Primary BAs are produced from cholesterol in the liver. Most of them are conjugated with taurine and glycine, and stored in the gallbladder and secreted into the duodenum, digest lipids and fatsoluble vitamins after meal stimulation. A study found that BAs were nearly twofold elevated in T2DM patients relative to healthy subjects, and the disproportion in BAs was associated with insulin resistance, ${ }^{31}$ which is consistent with our
GDM-resistance subtype. Earlier age at menarche also found to be a risk factor for GDM, ${ }^{17,32}$ however, another study uncovered that the associations between earlier age at menarche and the risk of GDM may be mediated by insulin resistance caused by overweight/obesity. ${ }^{33}$ Lee et al $^{15}$ revealed that the risk of developing GDM was significantly increased in participants with NAFLD, and this relationship remained significant after adjusted by metabolic risk factors and insulin resistance. These results revealed that other mechanisms might be involved in the process besides insulin resistance and obesity. For instance, pro-inflammatory cytokines from other visceral tissues could transfer to the liver, then lead to NAFLD. ${ }^{34}$

Enquobahrie et $\mathrm{a}^{35}$ found that the increased level of TG at the 1st trimester was a risk factor for GDM. We also observed this phenomenon in the GDM-resistance and GDM-mixed groups. However, this association weakened after adjusted by pre-BMI and GWG for GDM-resistance group. It suggests that the elevated TG level as a risk factor for GDM resistance group may mediated by obesity. Meanwhile, the combination of abnormal glucose metabolism and insulin resistance may cause more serious lipid metabolism disorder for the GDM-mixed group, which may explain the increased TG level in the 1st trimester is still a risk factor for this group before and after adjustment.

In our study, family history of diabetes is a risk factor for all GDM subtypes, and many researches have shown the same results in both obese and non-obese pregnant women. ${ }^{36,37}$ The following reasons might explain the relationship between family history of diabetes and GDM. Maternal diabetes during pregnancy lead to a transgenerational transmission of diabetes risk. ${ }^{38}$ Meanwhile, the cumulative incidence of type 2 diabetes was significantly increased in the first 5 years after the delivery of GDM women. ${ }^{39}$ At the same time, diabetes is a kind of polygenetic disease with overlaps of many susceptible genes of GDM. ${ }^{40,41}$

Many studies considered the increasing level and cutoff points of FBS in early pregnancy as a risk factor for GDM, but their results are inconsistent. ${ }^{42-44}$ The areas and ethnic variations among participants, and the different diagnostic criteria for GDM may explain the reasons for these inconsistencies. In this study, the association between the elevated level of FBG in the first trimester and GDM might be relevant to the high-carbohydrate diet of women in the northwest of China.

Limitations of our study included that this is a singlecenter study, which is restricted for further stratified analysis of different regions. Furthermore, the absence of data 
on pre-pregnancy islet $\beta$-cell function prevented us from presenting the dynamic characteristics of glucose metabolism. Finally, we did not collect the blood sample to examine the genetic loci of GDM subtypes in this study, which we will focus in the future.

In conclusion, our study showed that women with GDM exhibited heterogeneity based on glycemic physiology. The risk factors of GDM were different for each subtype, and the effect of many obesity-related risk factors on GDM was mediated by pre-pregnancy overweight/obesity and the elevated GWG during the first-trimester. Our study partly explained the reason for the inconsistency of risk factors on GDM about current studies. With the deep knowledge of etiology and pathogenesis on GDM subtypes, we will have a deeper understanding of the discrepancy on risk factors of GDM.

\section{Data Sharing Statement}

We would like to share the clinical data of our participants. Please contact Wei Cui through doctorweiweixjtu@126.com.

\section{Ethics Approval}

This study was approved and supervised by the Ethics Committee of the Xi'an Jiaotong University First Affiliated Hospital, and it was reviewed and recorded by the Northwest Women and Children's Hospital. All participants signed informed consent form, and the study was performed according to the Declaration of Helsinki, with a clinical trial registration number of ChiCTR 1900026735.

\section{Acknowledgments}

We thank all obstetricians of the Northwest Women and Children's Hospital who participated in the study and contributed to the collection of data.

\section{Author Contributions}

All authors made substantial contributions to conception and design, acquisition of data, or analysis and interpretation of data; took part in drafting the article or revising it critically for important intellectual content; agreed to submit to the current journal; gave final approval of the version to be published; and agree to be accountable for all aspects of the work.

\section{Funding}

This work was supported by the Natural Science Foundation of Shaanxi Province (No. 2020GXLH-Y-029,
2019JQ069, 2019JM262), the Bethune-Merck Diabetes Research Foundation (No. G-X-2019-056), the Clinical Research Award of the First Affiliated Hospital of Xi'an Jiaotong University, China (No. XJTU1AF-CRF-2019007), the Natural Science Foundation of China (No. 81801459; No. 81741079; No. 82071732), the Natural Science Foundation for Postdoctoral Scientists of China (No. 2018M641001, No. 2016M600799), the Fundamental Research Funds for the Central University (No. Z20186124, Z201704129).

\section{Disclosure}

The authors declare that they have no known competing financial interests or personal relationships and conflicts of interest that could have appeared to influence the work reported in this paper.

\section{References}

1. American Diabetes A. 2. Classification and diagnosis of diabetes: standards of medical care in diabetes-2020. Diabetes Care. 2020;43 (Suppl 1):S14-S31. doi:10.2337/dc20-S002.

2. Spaight C, Gross J, Horsch A, Puder JJ. Gestational diabetes mellitus. Endocr Dev. 2016;31:163-178. doi:10.1159/000439413

3. Kahn SE, Prigeon RL, McCulloch DK, et al. Quantification of the relationship between insulin sensitivity and beta-cell function in human subjects. Evidence for a hyperbolic function. Diabetes. 1993;42(11):1663-1672. doi:10.2337/diab.42.11.1663

4. Powe CE, Allard C, Battista MC, et al. Heterogeneous contribution of insulin sensitivity and secretion defects to gestational diabetes mellitus. Diabetes Care. 2016;39(6):1052-1055. doi:10.2337/dc152672

5. Santos S, Voerman E, Amiano P, et al. Impact of maternal body mass index and gestational weight gain on pregnancy complications: an individual participant data meta-analysis of European, North American and Australian cohorts. BJOG. 2019;126(8):984-995. doi:10.1111/1471-0528.15661

6. Shah A, Stotland NE, Cheng YW, Ramos GA, Caughey AB. The association between body mass index and gestational diabetes mellitus varies by race/ethnicity. Am J Perinatol. 2011;28(7):515-520. doi:10.1055/s-0031-1272968

7. Zhang C, Rawal S, Chong YS. Risk factors for gestational diabetes: is prevention possible? Diabetologia. 2016;59(7):1385-1390. doi:10.1007/s00125-016-3979-3

8. Jin WY, Lin SL, Hou RL, et al. Associations between maternal lipid profile and pregnancy complications and perinatal outcomes: a population-based study from China. BMC Pregnancy Childbirth. 2016;16(1):60. doi:10.1186/s12884-016-0852-9

9. Li G, Kong L, Zhang L, et al. Early pregnancy maternal lipid profiles and the risk of gestational diabetes mellitus stratified for body mass index. Reprod Sci. 2015;22(6):712-717. doi:10.1177/ 1933719114557896

10. Wang N, Song L, Sun B, et al. Contribution of gestational diabetes mellitus heterogeneity and prepregnancy body mass index to large-for-gestational-age infants-A retrospective case-control study. J Diabetes. 2020. doi:10.1111/1753-0407.13113

11. Retnakaran R, Hanley AJ, Raif N, Connelly PW, Sermer M, Zinman B. C-reactive protein and gestational diabetes: the central role of maternal obesity. J Clin Endocrinol Metab. 2003;88 (8):3507-3512. doi:10.1210/jc.2003-030186 
12. Huvinen E, Grotenfelt NE, Eriksson JG, et al. Heterogeneity of maternal characteristics and impact on gestational diabetes (GDM) risk-Implications for universal GDM screening? Ann Med. 2016;48 (1-2):52-58. doi:10.3109/07853890.2015.1131328

13. Kong M, Lu Z, Zhong C, et al. A higher level of total bile acid in early mid-pregnancy is associated with an increased risk of gestational diabetes mellitus: a prospective cohort study in Wuhan, China. $J$ Endocrinol Invest. 2020;43(8):1097-1103. doi:10.1007/s40618020-01196-7

14. Bowers KA, Olsen SF, Bao W, Halldorsson TI, Strøm M, Zhang C. Plasma concentrations of ferritin in early pregnancy are associated with risk of gestational diabetes mellitus in women in the Danish National Birth Cohort. J Nutr. 2016;146(9):1756-1761. doi:10.3945/ jn.115.227793

15. Lee SM, Kwak SH, Koo JN, et al. Non-alcoholic fatty liver disease in the first trimester and subsequent development of gestational diabetes mellitus. Diabetologia. 2019;62(2):238-248. doi:10.1007/s00125018-4779-8

16. Karakosta P, Alegakis D, Georgiou V, et al. Thyroid dysfunction and autoantibodies in early pregnancy are associated with increased risk of gestational diabetes and adverse birth outcomes. J Clin Endocrinol Metab. 2012;97(12):4464-4472. doi:10.1210/jc.2012-2540

17. Wang L, Yan B, Shi X, et al. Age at menarche and risk of gestational diabetes mellitus: a population-based study in Xiamen, China. $B M C$ Pregnancy Childbirth. 2019;19(1):138. doi:10.1186/s12884-0192287-6

18. Xia J, Song Y, Rawal S, et al. Vitamin D status during pregnancy and the risk of gestational diabetes mellitus: a longitudinal study in a multiracial cohort. Diabetes Obes Metab. 2019;21(8):1895-1905. doi:10.1111/dom. 13748

19. American Diabetes Association. Executive summary: standards of medical care in diabetes-2013. Diabetes Care. 2013;36 Suppl 1:S410. doi: $10.2337 / \mathrm{dc} 13-\mathrm{S} 004$

20. Chen C, Lu FC, Department of Disease Control Ministry of Health PRC. The guidelines for prevention and control of overweight and obesity in Chinese adults. Biomed Environ Sci. 2004;17 Suppl:1-36.

21. Matsuda M, DeFronzo RA. Insulin sensitivity indices obtained from oral glucose tolerance testing: comparison with the euglycemic insulin clamp. Diabetes Care. 1999;22(9):1462-1470. doi:10.2337/ diacare.22.9.1462

22. Stumvoll M, Mitrakou A, Pimenta W, et al. Use of the oral glucose tolerance test to assess insulin release and insulin sensitivity. Diabetes Care. 2000;23(3):295-301. doi:10.2337/diacare.23.3.295

23. Stumvoll M, Van Haeften T, Fritsche A, Gerich J. Oral glucose tolerance test indexes for insulin sensitivity and secretion based on various availabilities of sampling times. Diabetes Care. 2001;24 (4):796-797. doi:10.2337/diacare.24.4.796

24. Kim SY, England L, Wilson HG, Bish C, Satten GA, Dietz P. Percentage of gestational diabetes mellitus attributable to overweight and obesity. Am J Public Health. 2010;100(6):1047-1052. doi:10.2105/AJPH.2009.172890

25. Yen IW, Lee CN, Lin MW, et al. Overweight and obesity are associated with clustering of metabolic risk factors in early pregnancy and the risk of GDM. PLoS One. 2019;14(12):e0225978. doi:10.1371/ journal.pone.0225978

26. Leng J, Zhang C, Wang P, et al. Plasma levels of alanine aminotransferase in the first trimester identify high risk Chinese women for gestational diabetes. Sci Rep. 2016;6(1):27291. doi:10.1038/ srep27291

27. O'Malley EG, Reynolds CME, Killalea A, O'Kelly R, Sheehan SR, Turner MJ. Maternal obesity and dyslipidemia associated with gestational diabetes mellitus (GDM). Eur J Obstet Gynecol Reprod Biol. 2020;246:67-71. doi:10.1016/j.ejogrb.2020.01.007
28. Hou W, Meng X, Zhao W, et al. Elevated first-trimester total bile acid is associated with the risk of subsequent gestational diabetes. Sci Rep. 2016;6(1):34070. doi:10.1038/srep34070

29. Kawada T. Gestational diabetes and dyslipidemia: a causal association. Eur J Obstet Gynecol Reprod Biol. 2020;251:277-278. doi:10.1016/j.ejogrb.2020.04.043

30. Tiikkainen M, Bergholm R, Vehkavaara S, et al. Effects of identical weight loss on body composition and features of insulin resistance in obese women with high and low liver fat content. Diabetes. 2003;52 (3):701-707. doi:10.2337/diabetes.52.3.701

31. Catoi AF, Parvu A, Muresan A, Busetto L. Metabolic mechanisms in obesity and Type 2 diabetes: insights from bariatric/metabolic surgery. Obes Facts. 2015;8(6):350-363. doi:10.1159/000441259

32. Sun X, Yang L, Pan J, et al. Age at menarche and the risk of gestational diabetes mellitus: a systematic review and meta-analysis. Endocrine. 2018;61(2):204-209. doi:10.1007/s12020-018-1581-9

33. Petry CJ, Ong KK, Hughes IA, Acerini CL, Dunger DB. The association between age at menarche and later risk of gestational diabetes is mediated by insulin resistance. Acta Diabetol. 2018;55 (8):853-859. doi:10.1007/s00592-018-1162-7

34. Despres JP. Abdominal obesity as important component of insulin-resistance syndrome. Nutrition. 1993;9(5):452-459.

35. Enquobahrie DA, Williams MA, Qiu C, Luthy DA. Early pregnancy lipid concentrations and the risk of gestational diabetes mellitus. Diabetes Res Clin Pract. 2005;70(2):134-142. doi:10.1016/j. diabres.2005.03.022

36. Huvinen E, Eriksson JG, Stach-Lempinen B, Tiitinen A, Koivusalo SB. Heterogeneity of gestational diabetes (GDM) and challenges in developing a GDM risk score. Acta Diabetol. 2018;55 (12):1251-1259. doi:10.1007/s00592-018-1224-x

37. White SL, Lawlor DA, Briley AL, et al. Early antenatal prediction of gestational diabetes in obese women: development of prediction tools for targeted intervention. PLoS One. 2016;11(12):e0167846. doi:10.1371/journal.pone.0167846

38. Kamana KC, Shakya S, Zhang H. Gestational diabetes mellitus and macrosomia: a literature review. Ann Nutr Metab. 2015;66(Suppl 2):14-20. doi:10.1159/000371628

39. Kim C, Newton KM, Knopp RH. Gestational diabetes and the incidence of type 2 diabetes: a systematic review. Diabetes Care. 2002;25(10):1862-1868. doi:10.2337/diacare.25.10.1862

40. Dereke J, Palmqvist S, Nilsson C, Landin-Olsson M, Hillman M. The prevalence and predictive value of the SLC30A8 R325W polymorphism and zinc transporter 8 autoantibodies in the development of GDM and postpartum type 1 diabetes. Endocrine. 2016;53 (3):740-746. doi:10.1007/s12020-016-0932-7

41. Lin Z, Wang Y, Zhang B, Jin Z. Association of type 2 diabetes susceptible genes GCKR, SLC30A8, and FTO polymorphisms with gestational diabetes mellitus risk: a meta-analysis. Endocrine. 2018;62(1):34-45. doi:10.1007/s12020-018-1651-z

42. Salman L, Arbib N, Borovich A, et al. The impact of first trimester fasting glucose level on adverse perinatal outcome. $J$ Perinatol. 2018;38(5):451-455. doi:10.1038/s41372-018-0045-7

43. Zheng T, Ye W, Wang X, et al. A simple model to predict risk of gestational diabetes mellitus from 8 to 20 weeks of gestation in Chinese women. BMC Pregnancy Childbirth. 2019;19(1):252. doi:10.1186/s12884-019-2374-8

44. Saeedi M, Hanson U, Simmons D, Fadl H. Characteristics of different risk factors and fasting plasma glucose for identifying GDM when using IADPSG criteria: a cross-sectional study. $B M C$ Pregnancy Childbirth. 2018;18(1):225. doi:10.1186/s12884-018$1875-1$ 


\section{Publish your work in this journal}

Diabetes, Metabolic Syndrome and Obesity: Targets and Therapy is an international, peer-reviewed open-access journal committed to the rapid publication of the latest laboratory and clinical findings in the fields of diabetes, metabolic syndrome and obesity research. Original research, review, case reports, hypothesis formation, expert opinion and commentaries are all considered for publication. The manuscript management system is completely online and includes a very quick and fair peer-review system, which is all easy to use. Visit http://www.dovepress.com/testimonials.php to read real quotes from published authors.

Submit your manuscript here: https://www.dovepress.com/diabetes-metabolic-syndrome-and-obesity-targets-and-therapy-journal 